\title{
RECUPERAR EL PATRIMONIO CULTURAL INTANGIBLE A TRAVÉS DE LAS BEBIDAS \\ ARTESANALES GENERANDO UNA \\ OPORTUNIDAD A LA COMUNIDAD \\ CAMPESINA ${ }^{21}$
}

Ángela Esperanza Orjuela González

Dilan Esneider Peña Rojas

Félix Eduardo Rodríguez Medina 22

Fundación Universitaria San Mateo

Resumen:

La investigación presenta un plan de negocio para la creación de una empresa de licores y cremas artesanales de frutas propias de origen del departamento de Cundinamarca que va dirigido a estratos tres a cuatro de Bogotá. Como objetivo central se busca generar un nuevo tipo de producto de licor artesanal a través de la maceración de frutas de origen de la provincia de Sumapaz, para la recuperación de patrimonio intangible en las bebidas alcohólicas que son costumbres ancestrales del departamento los objetivos específicos, es el dar a conocer los beneficios de los licores sin el exceso del consumo y hacer uso de las más amplias variedad de frutas de origen de la región, apoyando al campo y a su desarrollo con el fin de generar emprendimiento.

La metodología a utilizar es cuantitativo y cualitativa de carácter exploratorio y descriptivo por lo tanto hará uso de herramientas de diagnóstico, análisis, recopilación de información, entrevistas y encuestasen donde se verá evidenciado la participación activa de la población segmentada.

Finalmente, se espera obtener unos buenos licores artesanales a partir de frutas de origen de la provincia de Sumapaz y recupera el patrimonio inmaterial, generando un valor de identidad.

\footnotetext{
${ }^{21}$ Proyecto adscrito al grupo de investigación Patrimonio cultural gastronómico colombiano de la Facultad de Ciencias Administrativas y Afines, Fundación Universitaria San Mateo, COL0161121.

${ }^{22}$ Proyecto adscrito al grupo de investigación Patrimonio cultural gastronómico colombiano de la Facultad de Ciencias Administrativas y Afines, Fundación Universitaria San Mateo, COL0161121.
} 


\section{Palabras clave:}

bebidas alcohólicas, cultivos de frutas, cambio cultural.

\section{Abstract:}

The investigation presents a business plan for the creation of a company of liquors and handmade creams of fruits from the department of Cundinamarca that is aimed at strata three to four of Bogota. The main objective is to generate a new type of product of artisan liquor through the maceration of fruits of origin of the province of Sumapaz, for the recovery of intangible heritage in alcoholic beverages that are ancestral customs of the department. The specific objectives are to publicize the benefits of liquor without excess consumption and to make use of the widest variety of fruits of origin in the region, supporting the field and its development in order to generate entrepreneurship.

The methodology to be used is quantitative and qualitative of an exploratory and descriptive nature, therefore it will make use of diagnostic tools, analysis, information gathering, interviews and surveys where the active participation of the segmented population will be evident.

Finally, it is expected to obtain some good artisan liquors from fruits of origin of the province of Sumapaz and recover the intangible heritage, generating an identity value.

\section{Keywords:}

Alcoholic beverages, Fruit Crops, Cultural change, Intangible cultural heritage 


\section{Introducción}

La identidad es la que nos hace únicos; son las costumbres y tradiciones que se trasmiten a las generaciones futuras, para así mantener una identidad propia y diferenciar a cada territorio alrededor del mundo. En Colombia, según palabras del periodista Rolando Garzón, "las bebidas artesanales son consideradas un peligro para la salud humana" (2018). Adicional a ello, en cifras dadas por diversos estudios la producción de chicha, chirrinche, guarapo, licores del pacífico colombiano y el sabajón generan un flujo económico fiscal irregular para la nación. Por otro lado, su proceso de elaboración no cuenta, en algunos casos, con las normas sanitarias para la producción masiva de las mismas.

La presente investigación se hace con el fin de fortalecer los valores del patrimonio nacional inmaterial en la cual se evidencia la historia, el estilo y el símbolo que representan estas bebidas artesanales para el departamento de Cundinamarca y Colombia en general. Así mismo, busca el mejoramiento de los procesos de producción de las mismas.

Aurelio Iragorri, exministro de agricultura y desarrollo Rural, reconoció el esfuerzo del Departamento Administrativo Nacional de Estadísticas (DANE) con las cifras reveladas y sostuvo que estas son un insumo y herramienta importantes para el desarrollo del sector agropecuario en el país. Carolina Moncayo (2016), indica que tan solo en el 14\% de las tierras colombianas se cultivan frutas y en el $0.8 \%$ plantas aromáticas teniendo el país mayores capacidades por su diversidad de fauna, flora y minerales. En este sentido, el proyecto busca incentivar el cultivo de frutas propias de origen de la región para impulsar la producción de licores y cremas artesanales a nivel local, incentivando la productividad de la población rural de manera puntual en la provincia de Sumapaz en Silvania; para luego llegar a diversos sitios y así posicionarse en algunos bares y restaurantes como canales de distribución, garantizando alto nivel de calidad en los productos, brindando una gama de sabores, colores y aromas característicos de las frutas en destilación y manteniendo precios justos. 
Por medio del trabajo investigativo se pretende incentivar los hábitos de consumo de estos licores, de manera que brinde apoyo a la comunidad rural; así como al sostenimiento de la empresa que se busca crear. Este proyecto está sustentado en el marco de la ley 1506 del 2014, en donde se establece el certificado de las Buenas Prácticas de Manufactura (BPM), para la fabricación, elaboración, hidratación o envase de bebidas alcohólicas.

\section{Marco teórico}

La chicha y el guarapo son bebidas del pueblo colombiano desde tiempos antiguos. Es por ello que la investigación que se presenta, intenta un acercamiento hacia estas bebidas legendarias y que siguen vigentes en la actualidad, y cuya influencia contribuye en la construcción un país lleno de caracteres y fundamentos de una identidad heredada por los ancestros que habitaron el altiplano cundiboyacense (Gutiérrez-Quecano, 2019).

La chicha, así como otras bebidas artesanales de Colombia se llegaron a considerar "un problema social"; y es allí, en donde entra a participar la medicina y el Estado a finales del siglo XIX. Durante este periodo, se comienza a demonizar y patologizar la producción y el consumo masivo de esta bebida. Este hecho se puede constatar luego del Bogotazo ${ }^{23}$, cuando se emite una ley nacional en donde la producción masiva de esta bebida es prohibida. (Pohl y Rodríguez, 2018).

El primer científico que puso en marcha la investigación de esta bebida fue Liborio Zerda, médico conservador a finales del siglo XIX y ministro de instrucción pública durante la Regeneración. Zerda, realizó un estudio con su colega Josué Gómez en el año 1889 y establecieron las características en lo químico y lo patológico de la chicha. Afirma Pohl (2018, como se cita en Saade y Calvo, 2001); a lo largo de toda esta época los estudios realizados por Zerda será fundamento científico para decir que "la chicha es mala y embrutece a la población" (pp. 31-32).

\footnotetext{
${ }^{23}$ Se conoce a una serie de hechos posteriores a la muerte del caudillo liberal Jorge Eliécer Gaitán el 9 de abril de 1948.
} 
Un artículo publicado por Zerda menciona:

Que el proceso de fermentación de la chicha urbana como proceso masivo, sin condiciones de saneamiento, es peligrosa, porque el maíz se tenía que dejar reblandecer por muchos días en agua y durante este proceso lo que se va a llamar el masato se le adicionaba panela y se dejaba fermentar". Durante este proceso se producía una toxina la cual llamo "tomaína", que era parecida a la toxina que se producía en el cuerpo humano en descomposición el cual se llamaba "cadaverina" (Pohl y Rodríguez, 2018).

Es por ello que "el consumo de la chicha era similar a comer muerto" y por tal que, eso generaría procesos degenerativos físicos y mentales en la población capitalina y por lo tanto se podía entender como una enfermedad; esto motiva en defensa su idea de una nueva enfermedad que se va a llamar el chichismo (Pohl, 2018). En esta época, la población capitalina estuvo en constante cambios por las revueltas políticas de la época entre 1890-1940 y la toma del poder de los dos partidos más representativos de la época que era los conservadores y los liberales.

Seguidamente, aparece la figura de Jorge Bejarano, médico cirujano el cual ocupó el cargo de Ministro de Higiene sobre los años 30 del siglo pasado, y publicará el libro al que titula La derrota de un vicio en donde este personaje afirma lo dicho anteriormente por el doctor Zerda, "la chicha es mala y daña, esto en perjuicio de la población" (Bejarano, 1950).

En dicha publicación, se da el nombre del bebedor como "El enchichado", considerado por algunas personas de la época como un sujeto más cercano al simio que al ser humano, y dando el nombre a la bebida de un "tosigo (veneno) maldito" (Calvo y Saade, 1998, p. 13). De esta manera, se pretendía sacar del mercado y del consumo una tradición para imponer la llegada de la cervecería más famosa del país, Bavaria.

Posteriormente, similares tratamientos sufrieron otras bebidas y esto conllevó a la desaparición parcial de parte del patrimonio cultural inmaterial de di- 
ferentes regiones en Colombia. Aquí es donde juega papel importante el trabajo que se presenta, al continuar esfuerzos de recuperación y mejora de diverso tipo licores y cremas artesanales

\section{Materiales y métodos}

Se trabaja sobre diverso tipo de licores y cremas artesanales a través de la maceración de frutas de origen de la provincia de Sumapaz (uchuva, mora y fresa principalmente) y con las comunidades locales de Silvania que se dedican al cultivo de estos frutos. Se recurre a un diseño experimental que se aplicará de manera trasversal. De otra parte, se ha realizado trabajos de tipo descriptivo para conocer en detalle la forma en que la población de Cundinamarca puede identificarse con sus saberes propios y generar un ingreso económico para el desarrollo de la agricultura local con frutas de origen de la Provincia.

\section{Resultados}

Con la presente investigación se espera obtener una nueva línea de bebidas alcohólicas artesanales de frutas propias de origen de la región de Cundinamarca, con las siguientes frutas que son: la uchuva, mora y fresa. Se busca la generación de una nueva gama de sabores y aroma característico que contienen estas bebidas.

Por consiguiente, aportara mejoras de la base nutricional para el ser humano, desarrollando a la par una campaña de consumo responsable. Luego se implementará una página web en donde las personas podrán acceder a la información que recopila una historia de tradición en las bebidas tradicionales de la zona de estudio (Silvania), y de manera paralela, la recuperación del patrimonio cultural inmaterial de esta región de Cundinamarca y de esta manera, reforzar su identidad local y generar nuevas oportunidades económicas a la población local campesina como parte de actividades de Responsabilidad Social Empresarial. 


\section{Conclusión}

Sin haber dado termino al trabajo se puede adelantar que la producción de licores artesanales en zonas rurales de Cundinamarca, pueden ser recuperables basados en los conocimientos de las practicas que los ancestros cundiboyacense; en estas, se guardaba una riqueza invalorable, y que, al no conservarlas, registrarlas y desarrollarlas, se podría perder un patrimonio inmaterial local de toda esta región,

Por otro lado, se está comprobando que estas bebidas no son un peligro para la salud pública, sino el reflejo de una identidad regional, pero que como consecuencia de algunas investigaciones realizadas en siglo XIX estas se han demonizado, patologizado, en detrimento de su producción. 


\section{Referencias bibliográficas}

Bejarano, J. (1950). La derrota de un vicio. Bogotá: Editorial Queima.

Calvo, O. y Saade, M. (2002). La cuidad en cuarentena chicha patología social y profilaxis. Bogotá: Ministerio de Cultura.

Garzón, R. (2018). En chicha y otros tragos artesanales. Bogotá Colombia: Revista el tiempo. Recuperado de https://www.eltiempo.com/ economia/empresas/gasto-en-tragos-artesanales-en-colombia-229626

Gutiérrez-Quecano, R. (Comp.). (2019). Tendencias en los procesos de formación del gastrónomo en Colombia. Bogotá: Editorial Fundación Universitaria San Mateo. Recuperado de https:// palma.sanmateo.edu.co/
Moncayo, C. (2016). INSTITUTO NACIONAL DE CONTADORES PUBLICOS, Recuperado de https://www.incp.org.co/dane-presenta-las-cifras-reales-del-campo-colombiano/

Restrepo, C. (2012). Historia de la cocina y la gastronomía. Recuperado de https://www. historiacocina.com/es/historia-de-la-chicha 


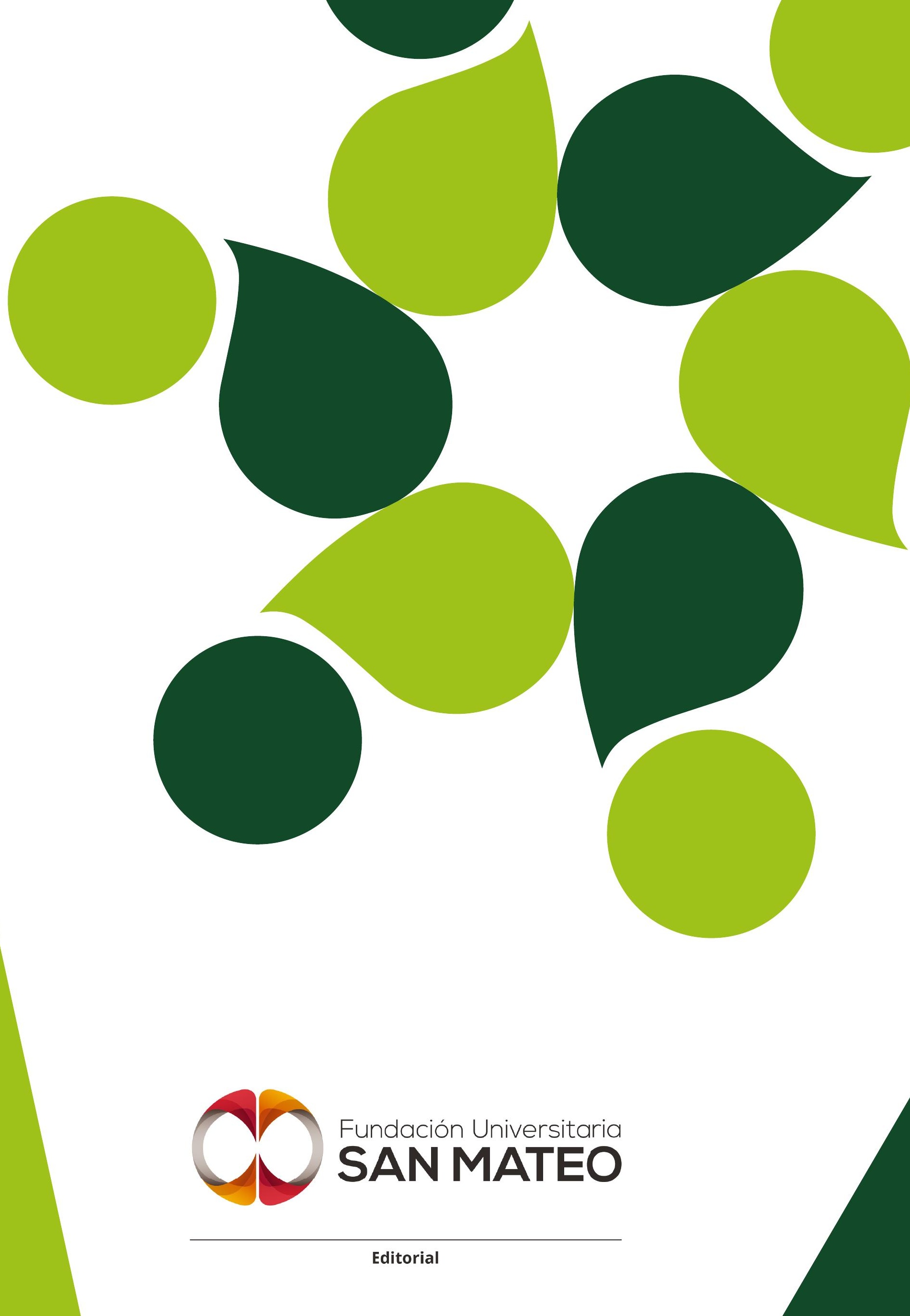

